\title{
Spectral discrimination of healthy and Ganoderma-infected oil palms from hyperspectral data.
}

\begin{abstract}
Although hyperspectral remote sensing has been used to study many agricultural phenomena such as crop stress and diseases, the potential use of this technique for detecting Ganoderma disease infestations and damage to oil palms under field conditions has not been explored to date. This research was conducted to investigate the feasibility of using a portable hyperspectral remote-sensing instrument to identify spectral differences between oil-palm leaves with and without Ganoderma infections. Reflectance spectra of samples representative of three classes of disease severity were collected. The most significant bands for spectral discrimination were selected from reflectance spectra and first derivatives of reflectance spectra. The significant wavelengths were identified using one-way analysis of variance. Then, a Jeffries-Matusita (JM) distance measurement was used to determine spectral separability between the classes. A maximum likelihood classifier method was used to classify the three classes based on the most significant wavelength spectral responses, and an error matrix was finally used to assess the accuracy of the classification.
\end{abstract}

Keyword: Hyperspectral; Oil palm; Ganoderma. 